\title{
Real-Time Control of Resonance in "Smart" Shape-Memory Alloy Hybrid Laminates
}

\author{
C.M. Friend and P.J. Armstrong \\ School of Engineering \& Applied Science, Cranfield University, Shrivenham Campus, Swindon, \\ Wiltshire SN6 8LA, U.K.
}

\begin{abstract}
Active Strain Energy Tuning (ASET) using Shape-Memory Actuators has been proposed for the vibration control of 'smart' composite structures. This paper presents preliminary experimental measurements on some simple prototype SMA hybrid 'Smart' structures. The results confirm previous modelling and show that ASET can indeed be used to control vibration characteristics. The most significant control mechanism appears to be realtime shifting of resonance frequency. This practical implementation also reveals additional factors which must be considered when designing such structures.
\end{abstract}

\section{INTRODUCTION}

In recent years a new technology has emerged in structural engineering. This is the advent of 'smart' structures. These react to their environment to produce controlled changes in defined structural parameters, such as vibration. 'Smart' structures essentially require three components (i) sensors - to detect changes in the structure's environment, (ii) signal processing, and (iii) actuators - which alter the performance of the structure in response to sensory data. In most 'smart' structural concepts the sensors and actuators are integrated within the structure itself, and many of the sensor and actuator materials are familiar from other sensing/actuation applications; for example Shape-Memory Alloys.

Low frequency noise poses a problem in many situations. The use of large structural panels, such as components in transport vehicles, buildings, etc., can result in significant noise problems since they often have natural frequencies below $100 \mathrm{~Hz}$. Particular problems also arise when these resonance frequencies lie within the infrasonic range (below 20Hz) since external excitation can couple with these low natural frequencies to increase the amplitude of vibration causing discomfort and fatigue. Well established protection systems such as ear muffs, active noise reduction, attenuating materials and insulators are not always feasible in many of these applications, or are inefficient in this frequency range. Active structural control using 'smart' structures therefore becomes attractive.

Vibration control of 'smart' structures has traditionally concentrated on high band-width, low strain piezoelectric actuators which damp the amplitude of vibration by applying anti-strain to the structure. However, with low frequency vibration there is an alternative control strategy employing lower bandwidth, high strain actuators. Amongst the actuator materials proposed for such concepts are ShapeMemory Alloys (SMAs). SMAs have been employed as actuators in 'smart' structures by either embedding them within, or surface mounting them to, composite components. To date work on such concepts has demonstrated the viability of vibration, shape, and damage control[1]. The use of 'smart' structures containing SMAs has been widely discussed, however, critical review of the literature shows that published work is not extensive, and heavily biased towards modelling. SMA hybrid composites have been investigated using essentially two approaches[1]. Active Property Tuning (APT) essentially exploits property changes within SMAs during actuation (such as their significant change in 'effective' modulus), and Active Strain Energy Tuning (ASET) exploits changes in the strain-energy balance within a host composite when a SMA actuates. ASET is particularly interesting since it appears to offer greater control authority over hybrid composite structures. 
When using ASET for vibration control, the approach is not to actively control amplitude, but to modify a composite's properties (such as resonance frequency) in real-time. Resonance frequencies can then be actively moved away from excitation present within the structure's environment. Modelling of these concepts [1] has shown that such vibration control is possible, however, there is only limited experimental verification $[1,2]$. This paper presents preliminary results from an experimental programme on ASET and highlights some of the practical aspects which contribute to the performance of these SMA hybrid composite structures.

\section{EXPERIMENTAL PROCEDURES}

The aim of this preliminary work was to experimentally investigate the vibration control of thin composite components. The work was conducted on glass-fibre reinforced epoxide panels (dimensions $600 \mathrm{~mm} \times 50 \mathrm{~mm} \times 2 \mathrm{~mm}$ ) which were designed to have resonance frequencies within the infrasonic range (slightly below $20 \mathrm{~Hz}$ ). The fabrication of such hybrid panels has been described elsewhere [3,4]. The panels were constructed by hand lay-up and vacuum-bagging using Fibredux 916 woven glassfibre/epoxide pre-preg. $\mathrm{Ni}$ - $\mathrm{Ti} \mathrm{SMA}$ actuators with a transformation temperature of $50^{\circ} \mathrm{C}$ were incorporated into these panels in the form of $300 \mu \mathrm{m}$ diameter wires. These were placed in the central planes of the panels to avoid macroscopic shape-change of the composites during actuation. The actuators lay parallel to the 0 direction of the $[0,90]$ symmetric laminates. Panels were constructed containing six actuators across their width, resulting in a total actuator volume fraction of $0.6 \%$. A oneway memory strain was initially introduced into the actuators by mechanically restraining the $\mathrm{Ni}$ - $\mathrm{Ti}$ wires in a custom-built jig. Composite panels were then built around the strained actuators. A $4 \%$ strain was maintained on the actuators during composite cure, which was carried out using a thermal cycle recommended by the pre-preg manufacturer for the vacuum-bag processing of the resin system. Once the cure cycle was complete the hybrid panels were removed from their restraining jigs prior to vibration testing. During vibration testing actuation was stimulated by resistance heating of the SMA, although the true drive variable was temperature. To allow the latter to be measured fine Chromel-Alumel thermocouples were incorporated into the panels during manufacture.

Vibration testing was carried out using a custom-built test rig which supported the panels as thin beams. Clamped boundary conditions were employed at each end of the beams since previous work $[3,4]$ has shown that delamination of the actuator/host composite interface takes place if actuators are not constrained at the free surfaces of the hybrid composites. The test rig was mounted on a servo-hydraulic vibration test machine which excited the panels over a frequency range $1-100 \mathrm{~Hz}$. Resonance frequencies were determined using piezoceramic accelerometers mounted on both the panel and test rig. The differential signal between these two accelerometers was monitored, allowing both the resonance frequencies and amplitude of vibration to be determined.

\section{RESULTS AND DISCUSSION}

Actuation of the SMA produced a significant effect on the vibration response of these composite panels. This could be seen qualitatively by holding an unactuated panel at its natural (first resonance) frequency and then actuating the SMA. At this fixed frequency, actuation resulted in almost complete 'damping' of vibration; a clear illustration of vibration control. However, ASET-based control is a multi-variable problem and cannot be optimised using such simple experiments; however dramatic they may appear. The changes in natural frequency and vibration amplitude depended on both the active actuator fraction and degree of actuation (proportional to the temperature to which the actuators were heated). In this paper no attempt is made to completely characterise this multi-dimensional control space. Instead, preliminary results will be presented which describe the behaviour in iso-fraction and isothermal sections through this space. This will illustrate (i) the practical feasibility of ASET-based vibration control, (ii) the principal factors affecting control, and (iii) other factors which become critical when operating these 'smart' hybrid composites in practice. 


\subsection{Degree of Actuation}

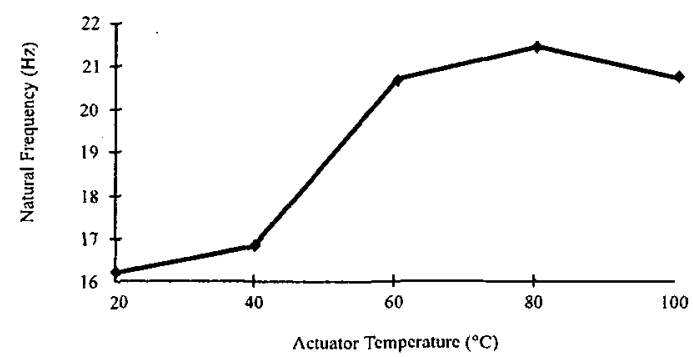

Figure 1: Effect of actuation temperature on natural frequency

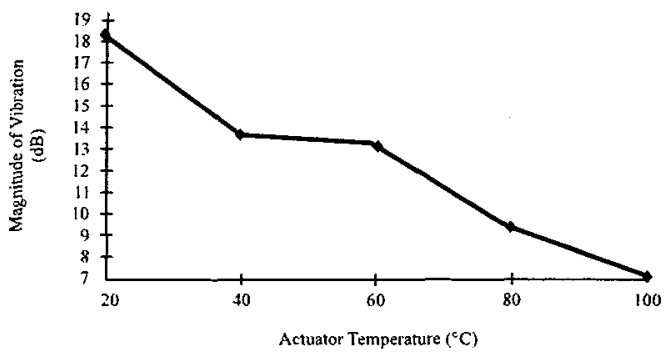

Figure 2: Effect of actuation temperature on magnitude of vibration

Figures 1 and 2 show iso-actuator fraction sections (six active actuators) through vibration response/ temperature/ actuator-fraction space. These illustrate the effect of actuation temperature on the natural frequency and magnitude of vibration (expressed in terms of accelerations) at this frequency. Figure 1 illustrates the authority which actuation exerted over the natural frequency at temperatures above $40^{\circ} \mathrm{C}$. Such a discontinuous response is not entirely unexpected since ASET, by implication, requires changes in internal strain energy within the host composite which results from the SMA actuation strain. The Ni-Ti employed in these panels had an actuation temperature of approximately $50^{\circ} \mathrm{C}$. No effect would therefore be expected below this temperature. The response following actuation was significant, resulting in typically $4 \mathrm{~Hz}$ increases in natural frequency over a narrow actuation range. There was little advantage in thermal excursions above $80^{\circ} \mathrm{C}$ which is consistent with the SMA reaching thermoelastic equilibrium between actuation temperature, actuation strain and internal stress. The natural frequency changes observed seem consistent with ASET and its reported effect on resonance frequency [1].

Figure 2 shows the effect of actuation temperature on the magnitude of vibration at these natural frequencies. There was a clear reduction in the magnitude of vibration with increasing temperature. Such a reduction must also have contributed to the vibration control observed in the qualitative demonstration described earlier. However, figure 2 gives little indication whether such effects were due to ASET or simply due to temperature rises within the experimental panels, resulting from heat-dissipation from the actuators.

\subsection{Actuator Fraction}

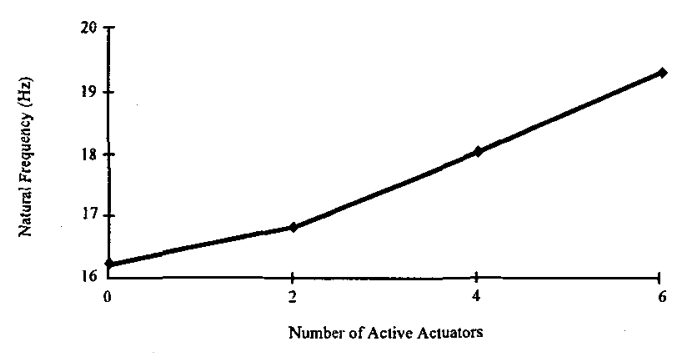

Figure 3: Effect of active actuator fraction on natural frequency

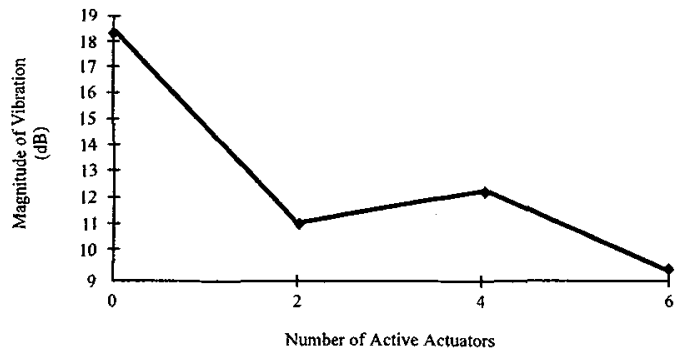

Figure 4: Effect of active actuator fraction on magnitude of vibration 
Figures 3 and 4 show typical isothermal sections (actuator temperature $50^{\circ} \mathrm{C}$ ) through vibration response/ actuation fraction/ temperature space. Figure 3 illustrates the effect of active actuator fraction on the natural frequencies of the hybrid panels. This isothermal section is at the actuation temperature of the $\mathrm{Ni}$ Ti SMA. It is clear from figure 3 that increasing the number of active actuators resulted in a monotonic increase in natural frequency, with a maximum shift of $3 \mathrm{~Hz}$ when all six actuators were employed. This observation is again, not entirely unexpected since ASET-based vibration control depends on the introduction of internal strain via the SMA actuators. Larger numbers of active actuators are capable of introducing higher levels of internal strain since each is operating under a lower effective applied stress, and would therefore be expected to produce larger shifts in natural frequency. In this sense changes in actuator fraction (at constant actuator temperature) operates in a similar manner to increases in actuator temperature (for a fixed fraction of actuator - figure 1). The magnitude of the frequency shifts in figures 1 and 3 are also consistent with those observed in the limited data available from the literature [1].

Figure 4 shows the effect of active actuator fraction on the magnitude of vibration at the natural frequencies. There was a clear reduction in the magnitude of vibration. However, in this case the reduction was not due to thermal effects within the panels alone, but to the effect of increasing actuation force and its influence on resonance through ASET.

\subsection{ASET or Thermal Effects?}

Most observations in this preliminary study appear to support the hypothesis that ASET has a significant influence on vibration response. This is particularly clear from the effect on natural frequency (figures $1 \& 3$ ). However, it is less clear whether ASET has a similar effect on the magnitude of vibration since figure 2 suggests that actuator temperature may play a more significant role in controlling damping. The latter effect was most likely due to the influence of temperature on the mechanical properties of the host epoxide composite. Such thermal effects can be separated from ASET by measuring the effect of temperature on similar experimental panels containing no actuators, but with similar sized embedded heating elements. The results from such experiments are contained in figures 5 and 6.

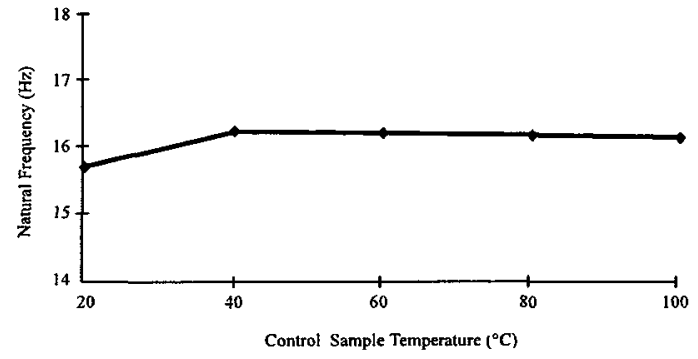

Figure 5: Effect of temperature on natural frequency

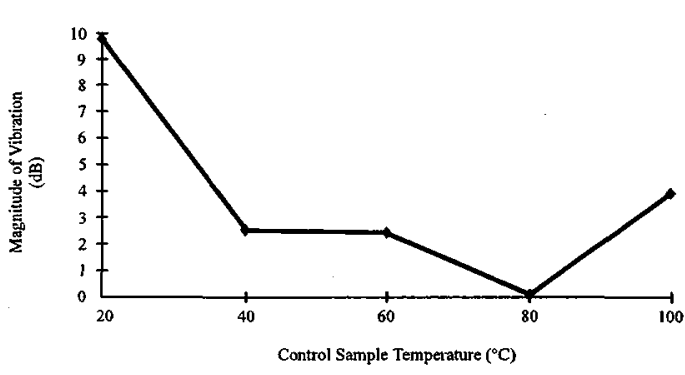

Figure 6: Effect of temperature on magnitude of vibration

Figure 5 shows the effect of temperature on the natural frequency of these 'control' panels. The base-line frequencies were slightly lower than the SMA hybrid composites since it was difficult to match the moduli of the actuators and heating elements. However, it is clear from figure 5 that temperature had little effect on natural frequency. This confirms previous observations that ASET was the principal mechanism controlling natural frequency. Figure 6 however, shows the effect of temperature on the magnitude of vibration at the natural frequency. There was considerable damping with increasing temperature. It is therefore clear that a large proportion of the damping observed in this programme resulted from changes in the mechanical properties of the host composite due to heat dissipation from the actuators. However, figure 4 shows that the damping observed in the hybrid panels was not completely due to this process and that ASET plays some role in controlling the magnitude of vibration. 


\section{CONCLUSIONS}

1 Active Strain Energy Tuning using Shape-Memory Actuators can be used practically to control the vibration characteristics of 'smart' composite structures.

2 The most significant. vibration control mechanism appears to be the real-time shift of natural frequency.

3 Significant and useable frequency shifts can be achieved with very small actuator fractions.

4 Optimisation of these shifts is a complex multivariable problem. At this preliminary stage it appears that higher actuator fractions and actuator drives are the most technologically useable combinations of these variables.

5 This practical implementation has also revealed additional factors which must be considered in designing and constructing SMA hybrid structures. The most important of these is heat dissipation from the actuators which has a significant effect on vibration control. Such practical observations contrast sharply with prior modelling.

\section{References}

[1] Laing, J. Jia, C. Rogers, Proc. 30th AIAA, ASME/ASCE/AHS/ASC Structures, Structural Dynamics, and Materials Conf. (AIAA, 1989) pp. 1504-1513.

[2] J-E Bidaux, N. Bernet, C. Sarwa, J-AE Manson and R. Gotthardt, proc ICOMAT '95, Lausanne (1995), J. de Physique IV, C8, Vol 5, pp. 1177-1182.

[3] C.M. Friend, N.B. Morgan and V. Wise, Materials for Smart Systems, MRS, Symp. Proc., Vol. 360, MRS, (1995), pp. 513-518.

[4] C.M. Friend and N.B. Morgan, Proc. ESOMAT `94, Barcelona, (1994). J. de Physique IV, C4, Vol 5, pp. 415-420. 